\title{
The hole at the bottom of the Moon
}

\section{A giant crater on the lunar farside holds the key to a catastrophic bombardment that reshaped the Moon, Earth and other \\ planets. Eric Hand reports.}

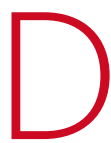

hofar 961 wasn't like the other Moon rocks. Looking at its freshly cut face, geochemist Randy Korotev noticed immediately how dark it was almost purple - and that it contained big metallic grains. It was so different from anything he'd seen before that he began to wonder. Was it from the 'big one'?

Korotev, of Washington University in St Louis, Missouri, already knew that Dhofar 961 was a piece of the Moon, chipped off by some anonymous impact so that it escaped the Moon's feeble gravitational grasp and succumbed to Earth's. Tens of thousands of years ago, Dhofar 961 fell into the Oman desert. A few years ago, it fell into the hands of collectors eager to make a buck. After fruitless searches on eBay, Korotev found a reputable dealer online selling a 6-gram piece of Dhofar 961 for US $\$ 1,000$ per gram -30 times more expensive than gold.

\section{"The Moon is a witness plate for what happened on the Earth." - David Kring} Korotev bought a sliver, and sacrificed a third of it for a chemical analysis that confirmed his suspicions.

Unlike the other 59 known lunar meteorites, which have chemical compositions that trace back to three specific regions on the Moon, Dhofar 961 probably hails from a fourth: a deep, dark hole at the bottom of the lunar backside, known as the South Pole-Aitken basin. It marks the site of the biggest-known blast the Moon has seen. And trapped within Dhofar 961 might be a record of that event, which would make it a clue to whether, and when, the inner Solar System endured a catastrophic pummelling in its youth.

Really large impacts such as this one leave not just craters but basins, deep and complex, their shock waves frozen into concentric rings like a bullseye. Even by basin standards, though, South Pole-Aitken is a doozy. Within the Solar System it is second in size only to Mars's 10,000kilometre-long Borealis basin, which as scientists report in this issue (see page 1212) was made by an impact so large that it seems to have sliced the top off Mars's northern hemisphere. South Pole-Aitken itself is more than 2,600 kilometres across and 12 kilometres deep, big enough to blot out half of China and hide the highest mountains of Tibet.

South Pole-Aitken is not only the biggest basin on the Moon, but also the oldest, based on the relative chronology that geologists piece together by mapping the way craters overlap each other. An absolute date for it is, however, unknown.

Just after the Solar System formed 4.6 billion years ago, leftover planetesimals regularly blasted the newborn planets. The barrage even knocked off enough of Earth to create the Moon in the first place. By 3.8 billion years ago, impact rates had tailed off to a level not too different from those of today (see graphic).
The south pole of the Moon, with a relief map of South Pole-

Aitken (purple area on the inset), mapped from the Clementine orbiter.

The question is what happened in between: did the impacts decrease smoothly, or was there, as many scientists suspect, a big spike 3.9 billion years ago? Given South Pole-Aitken's prominence at the bottom of the cratering heap, its age provides a crucial constraint on this 'late heavy bombardment' or 'lunar cataclysm'. An early date for South Pole-Aitken means a broader peak in the bombardment rate, or possibly a steady rate throughout the period. A later date speaks to cataclysm.

This is why the US National Academies last year called dating South Pole-Aitken the most important goal in lunar science. Date the basin, and you test the idea of a cataclysm, with 
"It's a major unsolved problem," says Jeffrey Taylor, a geologist at the University of Hawaii. "And the Moon is the only place we can address it."

\section{Dating the impacts}

Look at the Moon for even a moment, and it's clear that the place has been brutalized. Yet for many years, scientists thought that its cratered surface resulted from inner turmoil rather than outer. Impact craters were mistakenly identified as volcanic calderas, the remnants of explosive eruptions. "You have no idea how pervasive this idea was - that volcanics were responsible for everything on the Moon," says Don Wilhelms, a retired geologist who was the first to map the South Pole-Aitken basin in the early 1970s, when working at the US Geological Survey in Menlo Park, California. With Steve Squyres, Wilhelms was also the first to propose the existence of the Borealis basin on Mars ${ }^{1}$.

As the space race took off, rich new Moon maps were produced, and pioneering astrogeologists buried the volcanic theories (see page 1164). The big basins, they deduced, were all caused by impacts. Then, by the early 70s, the Apollo astronauts had brought back another surprise: Moon rocks that showed that the impacts were all roughly the same age. The huge Imbrium basin came in at an age of 3.85 billion years; nearby Nectaris, separated in the relative chronology by hundreds of substantial craters,

all its ensuing implications. It tells you what was happening on the Moon early on and, by inference, what was going on in the rest of the Solar System. It tells you whether the inner planets got smacked suddenly in an atmosphere-annihilating blast of impacts. And that has implications for the origins of life. Was the great bombardment so severe that it sterilized any life that had got started before then? Did it create the hellish conditions that many of the earliest life-forms seem to have endured? Could it even have moved life from one planet to the next, throwing travellers such as Dhofar 961 from surface to surface, complete with bacterial hitchhikers? was just 50 million years younger. Nothing was older than 4 billion years.

In 1973, Fouad Tera and his colleagues at the California Institute of Technology in Pasadena first used the term 'cataclysm' to explain the extreme pace of the impacts. "It must in any event have been quite a show from the Earth, assuming you had a really good bunker to watch from," they wrote in an abstract to that year's Lunar and Planetary Science Conference.

It wasn't just a 'show from the Earth', though; it was the greatest show the Earth itself has ever experienced - the sort of show you're lucky to come through intact. The Moon and Earth are so close to each other that whatever happened on the Moon also happened on Earth - and then some. The record has been lost on Earth because most impact craters are erased through weathering, erosion and the continuous churn of plate tectonics. That makes the Moon "a witness plate for what happened on the Earth," says David Kring, a geologist at the Lunar and Planetary Institute in Houston, Texas.

And what a bad time it was. To model what Earth went through, Kring scaled up what happened to the Moon by a factor of 13 to account for the fact that Earth is a much larger target ${ }^{2}$. Given Wilhelms' estimate of 15 major lunarimpact basins in the 50 million years between Nectaris and Imbrium, this meant to Kring's team that a projectile big enough to form a 20kilometre crater hit Earth every few thousand years. Every million years, something would come along big enough to make a 1,000-kilometre basin. Such impacts would have vaporized Earth's oceans and steam-sterilized the surface; Kring says an atmosphere of rock vapour could linger for thousands of years after the impact.

Here's the crazy part: Kring's estimate is, in fact, very conservative. Earth's strong gravity could attract impactors at a frequency as much as 500 times higher than the Moon would. Moreover, Kring does not include in his calculations the 30 other huge basins that, according to Wilhelms, were formed after South Pole-Aitken and before Nectaris. If South PoleAitken turns out not to be significantly older than

Nectaris, then the frequency of doomsday rocks hitting Earth rises yet higher.

What's more, Wilhelms' basin count - a baseline for many studies - is now old, 
and is conservative itself. Herbert Frey, at the Goddard Space Flight Center in Greenbelt, Maryland, recently finished a new hunt for basins, based on topographical data collected by the Clementine Moon orbiter. At the 2008 Lunar and Planetary Science Conference, Frey's team reported 92 basins bigger than 300 kilometres across - twice as many as Wilhelms. "We've grossly underestimated the actual flux of objects that hit the Moon," Frey says. "It means that the Earth was probably not a very good place to be 4 billion years ago."

Yet some astrobiologists say that a cataclysm may have catalysed the origin of life rather than snuffed it out. A stream of comets or asteroids hitting the planet would have brought foreign organic material to Earth. The bombardment might have pierced the crust, stirring up deep convective currents in the mantle in such a way as to establish early continental crust. And, although surface oceans might have been stripped away, subsurface water and heat could have nourished heatloving organisms. It's probably no coincidence that in phylogenetic trees of life, the roots of the three major branches - bacteria, archaea and eukaryotes - tend to be heat-loving ${ }^{3}$.

\section{Ultimate causes}

One thing the lunar rocks make clear is that the bombardment dropped off pretty quickly about 3.85 billion years ago. But what was doing the pummelling in the first place? Some have claimed it was simply the expected collisions of things left over from the formation of the Solar System, but there's no obvious way there would have been enough projectiles at the beginning to last as long as would be needed for that. Others say the cataclysm was a fresh spike of new bombardments, but what would have caused such an influx of new impactors? "Some people didn't like it because they couldn't think of a mechanism," says Wilhelms.

A few years ago, one possible explanation surfaced from researchers based in Brazil, the United States and in Nice, France. The team developed a dynamical model for the Solar System that explained why Uranus and Neptune circle the Sun farther out and more eccentrically than expected ${ }^{4}$. In their model (sometimes called the Nice model), they started the infant Solar System with Neptune's orbit inside that of Uranus, and let the clock run. Some 700 million years later, Jupiter and Saturn fell into an orbital pattern, and the resulting gravitational pull caused Uranus and Neptune to be kicked farther away from the Sun. That in turn disrupted a massive disk of icy comets in the Kuiper belt beyond Pluto, and sent them hurtling into the inner Solar System.

There's one problem with all this. The chemical composition of material within lunar craters, as well as their size distribution, matches nicely with asteroids, not comets - suggesting that asteroids were the main, or most recent, impactors during the bombardment. The Nice modellers have an answer for that: the changes in Jupiter's and Saturn's orbits may have also disrupted the asteroid belt between Mars and Jupiter. That could have been enough to send asteroids smashing into Earth, the Moon and more.

Korotev says he is now a believer in the lunar cataclysm, thanks in part to the Nice model. Other work is resolving his other long-standing problem with the cataclysm hypothesis:

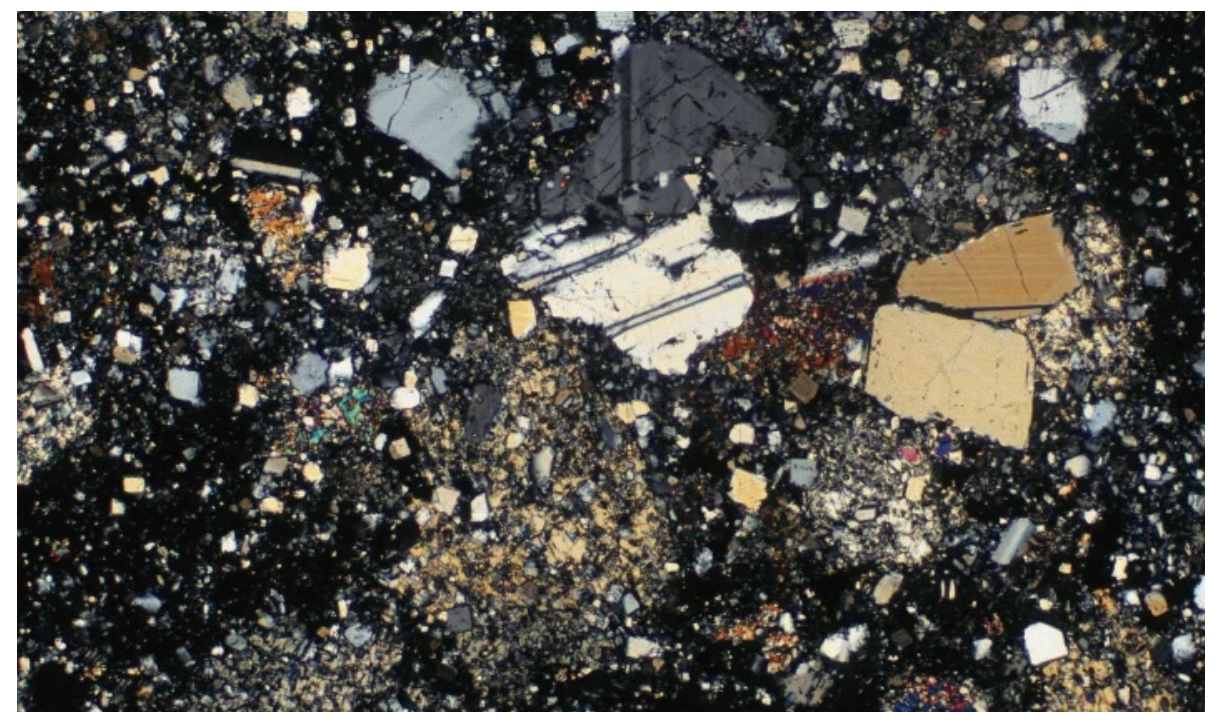

Time capsule: impact-melt Moon rocks, such as the slice shown here, all date from around the same time.

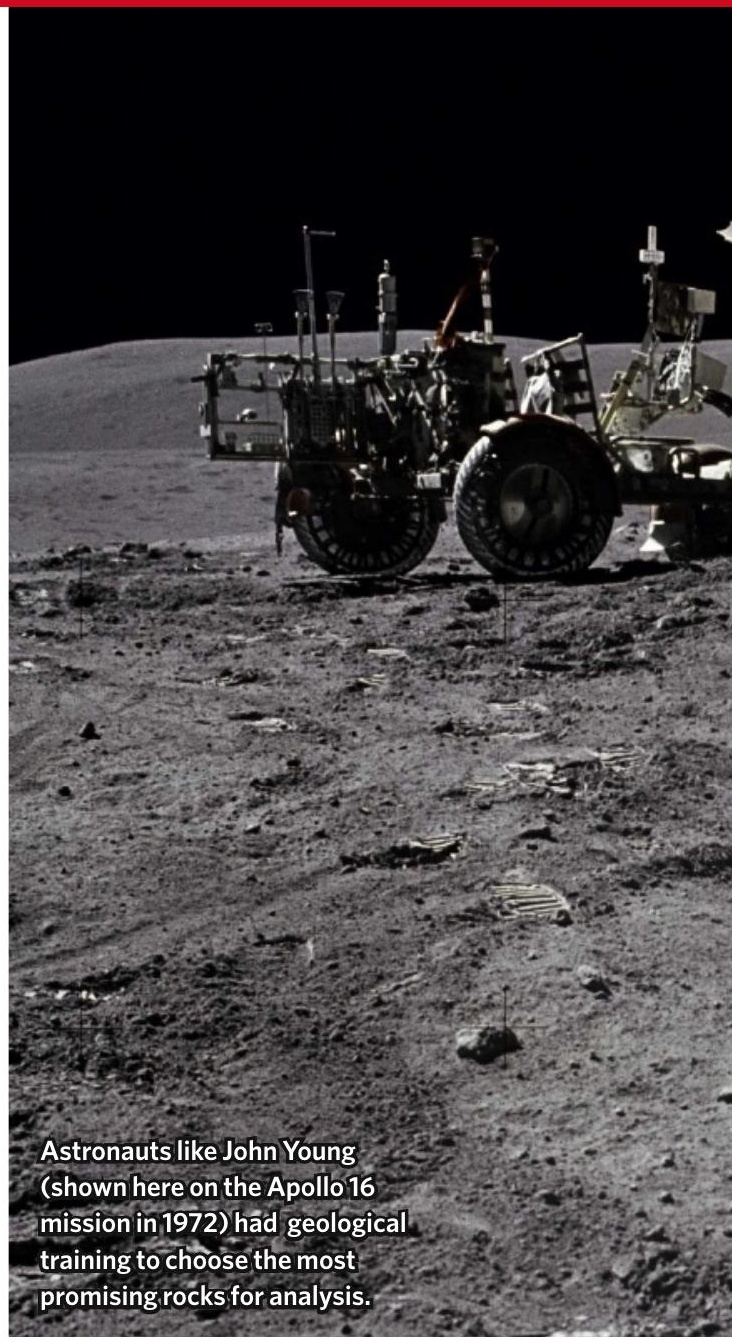

his belief that the dating of most Moon rocks to around 3.9 billion years ago is the result of an artificial selection bias. He and some other researchers have argued that the astronauts might have simply kept picking up rocks from the Imbrium impact over and over again, and that scientists interpreted them as being from different impacts.

Marc Norman, a cosmochemist at the Australian National University in Canberra, continues to wring new dates from the Apollo collection that may counter this challenge. Norman has been looking at impact melts, the recrystallized remains that contain an isotopic record of a rock being melted by an impact. In one Apollo rock, Norman dated 21 impact melts to within a 200-million-year window ${ }^{5}$. And he found that the melts fell into a number of age clusters, which he interprets as representing four different impact events. If his interpretation is correct, that would be more evidence that multiple big basins were formed within the narrow time period of the bombardment.

Other evidence is coming from meteorites, which unlike the geographically constrained Apollo rock collection are thought to have been hacked from all over the Moon. Working with Kring, Barbara Cohen, now at the Marshall Space Flight Center in Huntsville, Alabama, analysed four meteorites containing impact melts representing seven to nine impact events. None of the melts, she found, 


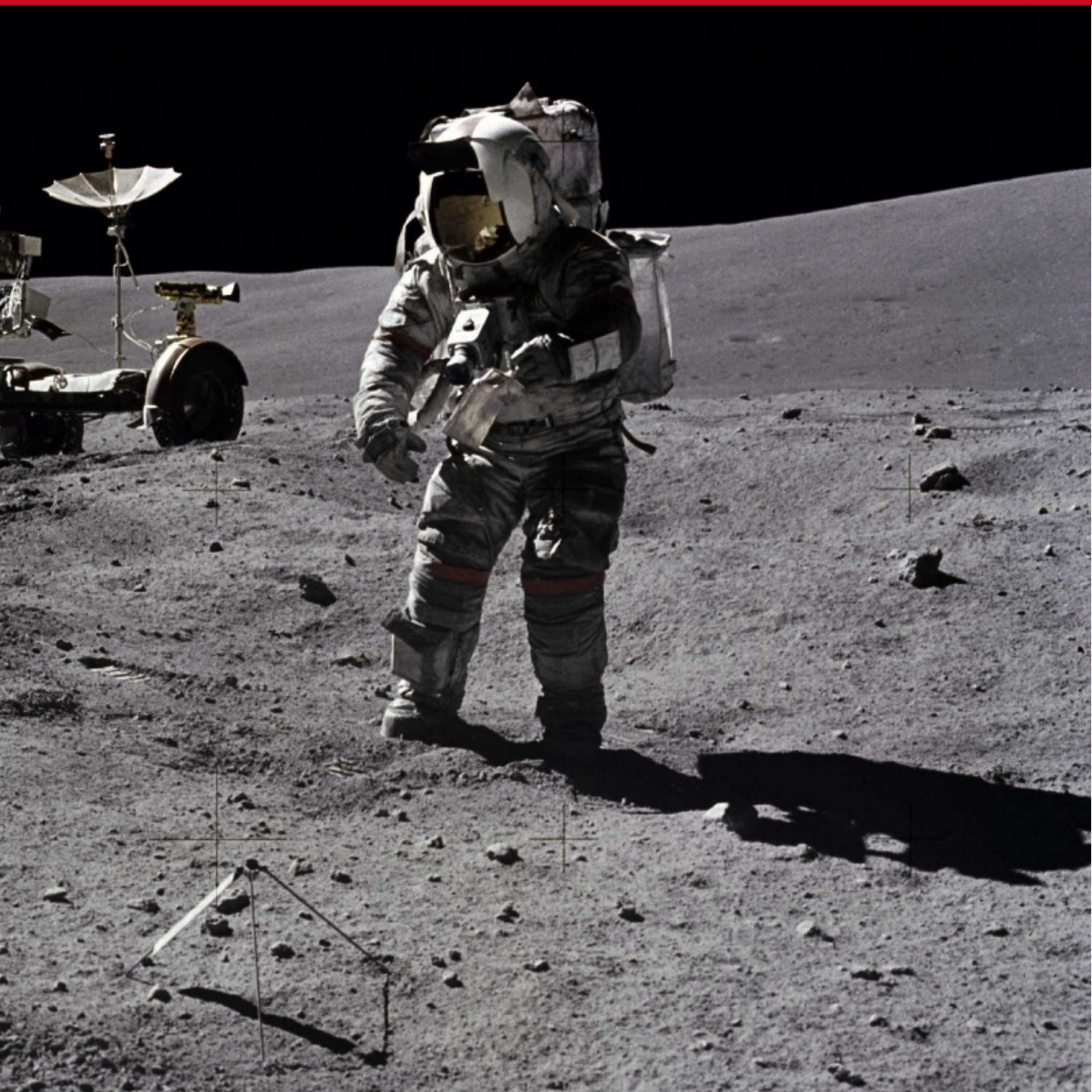

was older than 3.92 billion years ${ }^{6}$. Even the notorious martian meteorite Allan Hills 84001 - famous a decade ago for claims that it contained evidence of life - offers support for the lunar cataclysm theory: parts of the 4.5billion-year-old rock were altered in some sort of major event 3.9 billion years ago.

\section{Reaching for the Moon}

Still, some lunar scientists are not satisfied with Moon rocks fetched by astronauts or fallen from the sky. The best way to date South Pole-Aitken, they say, is to go there, get a rock, and date it.

Sending a simple robotic lander would be relatively cheap, but the robot's dating capabilities would not be good enough. Radioisotope dating requires a mass spectrometer, and one small enough to fly on a lander would have uncertainties of $10 \%$. On a 4-billion-year-old rock, that's 400 million years - exactly the sort of error that a mission travelling to date South Pole-Aitken is supposed to dispose of, not create. "You want to be able to say that your 4.2-billion-year-old age is different from a 4.0," explains Taylor. "It requires more accuracy than we have at present."

And so a group of lunar scientists is pushing for a South Pole-Aitken sample return mission, which would be the first lunar sample return since the last Soviet Luna spacecraft returned 170 grams of soil in 1976. The group, led by Brad Jolliff of Washington University in St Louis, plan to propose a mission for the next NASA New Frontiers competition, a mission class capped at $\$ 650$ million. NASA intends to start accepting proposals in December, with eventual selection in 2010 and a launch date no earlier than 2015.

In the most recent New Frontiers contest in 2005, a South Pole-Aitken mission called Moonrise made it to the final round but was ultimately bested by Juno, which is set to launch towards Jupiter in 2011. At the time, the risk of doing a sample return mission, with its many stages and components, was considered riskier than a simple orbiter such as Juno, says Jolliff, who proposal.

The old Moonrise project proposed two separate landers: one to go near the basin rim and the other, the centre, where impact melts are apt to be concentrated. Jolliff is leaving open the option to send just one lander to the basin centre. By 2020, if NASA's plans to return people to the Moon are realized, astronauts could already be encamped at the nearby Shackleton crater, placing them near the rim of the South Pole-Aitken basin. The Moonrise lander would collect rock and soil, and return to Earth with about a kilogram of material, Jolliff says.

Samples from a revamped Moonrise was the deputy principal investigator on that mission would allow lunar scientists to date many impact-melt crystals. The oldest, and most frequent, dates should correspond to the South Pole-Aitken impact. But other impactmelt dates would undoubtedly pollute the picture, as there are half a dozen other large basins within South Pole-Aitken. And debate would continue. "Landing in the middle of a field and getting a scoop of dirt is not going to give you the answer you need," says Norman.

In fact, Norman advocates both robotic and manned missions to the Moon, saying both are needed for a balanced exploration programme. "My feeling about sample return may be a little more nuanced than simply humans versus robots," he says. "Both can do the job provided we do the geologic homework, and neither will do an adequate job if we don't." Others, however, argue that the mystery of the great backside basin won't be solved until a human goes to the source and plucks a rock from within its blast shadow.

But returning people to the Moon will cost at least $\$ 230$ billion over two decades (according to the US Government Accountability Office), compared with the New Frontiers \$650-million cut-off. And Korotev thinks he can solve some of the mystery simply by dating the half-gram piece of the Moon he bought for $\$ 542$ (plus $\$ 12$ shipping and insurance). Later this year, he will share his precious sliver of South Pole-Aitken with Cohen. In Huntsville, she plans to sink a diamond-tipped drill bit into Dhofar 961 and extract several cores, each as fine as a human hair.

After vaporizing the samples with a laser, she will measure argon gas that has been trapped inside the rock crystal lattice for billions of years. Counting those atoms might allow her to count back in time to the blistering crucible of the bombardment. If she's successful, she will extract a date desired by lunar scientists for many moons - a big message from a little bottle.

Eric Hand covers physical sciences for Nature from the Washington DC office.

1. Wilhelms, D. E. \& Squyres, S. W. Nature $309,138-140$ (1989).

2. Kring D. \& Cohen, B. J. Geophys. Res. 107, 41-46 (2002).

3. Zahnle, K. \& Sleep, N. H. in Comets and the Origin of Life (eds Thomas, P., Chyba, C. \& McKay, C., eds) 175-208 (Springer, 1997).

4. Gomes, R. et al. Nature $435,466-469$ (2005)

5. Norman, M. D., Duncan, R. A. \& Huard J. J. Geochim. Cosmochim. Acta 70, 6032-6049 (2006)

6. Cohen, B. A., Swindle, T. D. \& Kring, D. A. et al. Science 290, 1754-1756 (2000).

See Editorial, page 1143, and News Feature, page 1164. 